\title{
Periodic solutions in general scalar non-autonomous models with delays
}

\author{
Pablo Amster and Lev Idels
}

\begin{abstract}
Theorems for the existence of periodic solutions for diverse models of population dynamics are obtained as corollaries of a few basic theorems, thus unifying the analysis of a broad class of scalar models in a single setting. The latter mechanism allows to obtain existence conditions for a broad class of nonlinear, non-autonomous models and models with state-dependent delays. The technique fulfills multiple roles: it can be used to expand on well-known results as well as to shorten existing proofs. We provide some examples which illustrate the applicability of our results.
\end{abstract}

Mathematics Subject Classification (2010) . Primary 34C25, 34G20;

Secondary 34K13, 34K05, 46B25.

Keywords. Continuation theorem, Leray-Schauder topological degree, Periodic solutions, Nonlinear non-autonomous delay differential equations, Time-varying and state-dependent delays, Existence, Population dynamics.

\section{Background and related results}

The problem of the existence of periodic solutions in population dynamics is a well-trodden venue, however, the results are scattered in the different sources, obtained via different mechanisms and are not unified. In the literature, we found (in historical order) the studies for general non-autonomous models [7, $10,14-16,18,22-24,28,31,34-37,39-43]$ with some applications for the particular models. The results obtained separately for some classical models described in the Appendix were presented in $[1-6,8,9,11,13,17,19-21,26,37,38]$.

Research partially supported by: PIP 11220090100637 CONICET, Project 20020090100067 UBACyT and a grant from Vancouver Island University. 
We begin with the most celebrated class of semilinear models:

$$
\frac{d x}{d t}=-a(t) x(t)+\lambda b(t) g(x(t-\tau(t))) .
$$

Most of the criteria involve the quantities $g_{0}=\lim _{u \rightarrow 0^{+}} \frac{g(t, u)}{u}$ and $g_{\infty}=$ $\lim _{u \rightarrow \infty} \frac{g(t, u)}{u}$; and the methods are based on Krasnoselskii fixed point theorem on cones in Banach spaces and its generalizations, e.g. Leggett-Williams theorem, and the use of the fixed point index.

Firstly, we quote some useful results. For (1.1), in [42] (2002) it was assumed that $a, b>0$ and $\tau$ are continuous and $T$-periodic, $g$ is continuous and positive, $\lambda>0$ and

$$
\bar{a}=\frac{1}{T} \int_{0}^{T} a(t) d t .
$$

The method of upper and lower solutions was used. In particular, [42, Theorem $5]$ it was shown that if $g$ is nondecreasing on $[0,+\infty)$ with $g(0)>0$ and $\frac{g(u)}{u} \rightarrow+\infty$ as $u \rightarrow+\infty$, then there exists a constant $\lambda_{*}>0$ such that the equation has a positive $T$-periodic solution for $0<\lambda<\lambda_{*}$, while there exists no such positive $T$-periodic solution if $\lambda>\lambda_{*}$. Under the same assumptions a similar result holds for the equation with reversed signs or a "mirror" equation

$$
\frac{d x}{d t}=a(t) x(t)-\lambda b(t) g(x(t-\tau(t))) .
$$

In [42] there are no explicit estimates (bounds) for $\lambda_{*}$ and no applications. Technically speaking, this paper was published in 2002, but all the results could be seen already in the earlier paper [10] (2001), where the basic assumptions are: $g, a, b, \tau$ are continuous and nonnegative; $a, b$ and $\tau$ are $T$-periodic functions; $a\left(t_{0}\right)>0$ for some $t_{0}$ in $[0, T]$. Under certain explicit conditions, intervals for $\lambda$ where the equation has one, two or no positive periodic solutions were obtained in [10]. All conditions for the existence of at least one/two positive periodic solutions, are expressed via different behaviors of the ratio $\frac{g(t, u)}{u}$, however, no applications are given.

For the special case of (1.1)

$$
\frac{d x}{d t}=-a(t) x(t)+g(t, x(t-\tau(t))),
$$

the existence of a positive periodic solution is guaranteed in [34, Theorem 2.1] (2004), if one of the following conditions holds:

$$
\left(H_{1}\right) \quad \liminf _{u \rightarrow 0} \min _{t \in[0, T]} \frac{g(t, u)}{a(t) u}>1, \quad \limsup _{u \rightarrow+\infty} \max _{t \in[0, T]} \frac{g(t, u)}{a(t) u}<1,
$$

or

$$
\left(H_{2}\right) \quad \limsup _{u \rightarrow 0} \max _{t \in[0, T]} \frac{g(t, u)}{a(t) u}<1, \quad \liminf _{u \rightarrow+\infty} \min _{t \in[0, T]} \frac{g(t, u)}{a(t) u}>1
$$


with applications to (4.8) and (4.10). However, for the models (4.5) and (4.11) the special condition $b(t)>a(t)$ for all $t$ is required. The following modification of the Eq. (1.2)

$$
\frac{d x}{d t}=-a(t) x(t)+g\left(t, x\left(t-\tau_{1}(t)\right), \ldots, x\left(t-\tau_{n}(t)\right)\right)
$$

was studied in [41] (2007) where the existence of single and multiple periodic solutions was obtained. For example [41, Theorem 3.3], the existence of a positive periodic solution is proven by conditions analogous to $\left(H_{1}\right)$ and $\left(H_{2}\right)$ in [34]. Multiple corollaries provide existence results for the biological models (4.2), (4.8) and (4.10) for which at least one positive periodic solution exists. It was also proven that for models (4.5) and (4.11) a positive periodic solution exists if $b(t)>a(t)$ for all $t$. Also, the existence of positive periodic solutions for the logistic model (4.1) with several delays was proven. In a related work [40] (2005) a slightly different form of model (1.3) is under study:

$$
\frac{d x}{d t}=-a(t) x(t)+g(t, u(t))
$$

where the vector $u$ is defined by

$$
u(t)=\left(x\left(\tau_{1}(t)\right), \ldots, x\left(\tau_{n-1}(t)\right), \int_{-\infty}^{t} k(t-s) x(s) d s\right) .
$$

As particular applications, the author claims the existence of periodic solutions for models (4.2), (4.8) and (4.10). For models (4.5) and (4.11) the special condition $\min _{0 \leq t \leq T} b(t)>\frac{1-H}{H^{2} T}$ is required, where $H=e^{-\int_{0}^{T} a(t) d t}$. The existence of positive periodic solutions for the logistic model (4.1) with several delays was proven as well (see also [41]).

In [36] (2004), the nonlinear model

$$
\frac{d x}{d t}=a(t) f(x(t)) x(t)-\lambda b(t) g(x(t-\tau(t))) .
$$

was examined. The existence, multiplicity and nonexistence of positive $T$-periodic solutions were proven, under the assumptions: $a, b, \tau$ are $T$-periodic functions, $\bar{a}>0, \bar{b}>0, f, g \in C([0,+\infty),[0,+\infty))$ with $g(u)>0$ for $u>$ $0,0<l \leq f(u)<L<+\infty, \lambda>0$. Explicit intervals for the parameter $\lambda$ such that equation has one, two or no positive $T$-periodic solutions were obtained, and no applications were given. All results obtained in [36] are applicable to a "mirror" equation. In the interesting paper [14] (2011) (see also [28]) the boundedness condition on $f$ was relaxed, and the existence of three periodic solutions under appropriate assumptions was obtained via the method of lower and upper solutions.

In this paper, we obtain existence results for certain class of abstract models. This, in turn, will lead to sufficient conditions for the existence of periodic solutions for diverse models originated from biological applications, unifying the analysis of a broad class of models of population dynamics in a single setting. It includes: nonlinear, non-autonomous models; models with multiple and state-dependent delays. Our technique is twofold: it can be used to expand on well-known existence results as well as to shorten existing proofs. We also 
study some qualitative properties of these solutions. We provide a variety of different examples, which shall explicate the applicability of our results.

\section{Main results}

\subsection{Continuation theorem for the abstract model}

We begin our study with an abstract existence theorem for the functional differential equation

$$
x^{\prime}(t)=\Phi(x)(t),
$$

where $\Phi$ maps the space of continuous $T$-periodic functions into itself. In more precise terms, set

$$
X:=\{x \in C(\mathbb{R}, \mathbb{R}): x(t+T)=x(t) \text { for all } t\}
$$

and assume that $\Phi: X \rightarrow X$ is continuous and maps bounded sets into bounded sets. For convenience, we define, for $r<s$,

$$
X_{r}^{s}:=\{x \in X: r<x(t)<s \text { for all } t\} .
$$

The closure of $X_{r}^{s}$ shall be denoted by $\operatorname{cl}\left(X_{r}^{s}\right)$. For $x \in X$, its absolute maximum and minimum values and its average $\frac{1}{T} \int_{0}^{T} x(t) d t$ are denoted by $x_{\max }$, $x_{\text {min }}$ and $\bar{x}$, respectively. If $U$ is an open and bounded subset of $X$ and $\mathcal{K}$ : $c l(U) \rightarrow X$ is compact with $\mathcal{K} u \neq u$ for all $u \in \partial U$, then the Leray-Schauder degree of the Fredholm operator $\mathcal{F}=I d-\mathcal{K}$ at 0 shall be denoted by $\operatorname{deg}_{L S}(\mathcal{F}, U, 0)$. For a detailed definition and properties of the degree see for example $[12,27]$; here, only the following basic properties shall be used:

1. (Solution) If $\operatorname{deg}_{L S}(\mathcal{F}, U, 0) \neq 0$, then $\mathcal{F}$ has at least one zero in $U$.

2. (Homotopy invariance) If $\mathcal{F}_{\lambda}=I d-\mathcal{K}_{\lambda}$ with $\mathcal{K}_{\lambda}: \operatorname{cl}(U) \rightarrow X$ compact such that $\mathcal{K}_{\lambda} u \neq u$ for all $u \in \partial U, \lambda \in[0,1]$ and $\mathcal{K}: \operatorname{cl}(U) \times[0,1] \rightarrow X$ given by $\mathcal{K}(u, \lambda):=\mathcal{K}_{\lambda}(u)$ continuous, then $\operatorname{deg}_{L S}\left(\mathcal{F}_{\lambda}, U, 0\right)$ does not depend on $\lambda$.

3. If $\mathcal{K}(\operatorname{cl}(U)) \subset V$, with $V \subset X$ a finite dimensional subspace, then

$$
\operatorname{deg}_{L S}(\mathcal{F}, U, 0)=\operatorname{deg}_{B}\left(\left.\mathcal{F}\right|_{c l(U) \cap V}, U \cap V, 0\right),
$$

where $\operatorname{deg}_{B}$ denotes Brouwer's degree.

4. If $\phi:[r, s] \rightarrow \mathbb{R}$ is continuous and $\phi(r), \phi(s) \neq 0$, then

$$
\operatorname{deg}_{B}(\phi,(r, s), 0)=\frac{\operatorname{sgn}(\phi(s))-\operatorname{sgn}(\phi(r))}{2} .
$$

Finally, we consider the natural inclusion $\mathbb{R} \subset X$ and define a mapping $\phi: \mathbb{R} \rightarrow \mathbb{R}$ as follows. For $\gamma \in \mathbb{R}$, let $x_{\gamma} \in X$ be the constant function given by $x_{\gamma}(t)=\gamma$ for all $t$; thus $\Phi\left(x_{\gamma}\right)$ is an element of $X$ and we may set

$$
\phi(\gamma):=\overline{\Phi\left(x_{\gamma}\right)}=\frac{1}{T} \int_{0}^{T} \Phi\left(x_{\gamma}\right)(t) d t .
$$

In the sequel, we shall ignore the isomorphism $\gamma \mapsto x_{\gamma}$; hence, the same symbol $\gamma$ shall be used to denote both a real number and the constant function $x_{\gamma} \equiv \gamma$. 
We establish the following continuation theorem, that will be the key for the further studies.

Theorem 2.1. Assume there exist constants $r<s$ such that

1. If $x^{\prime}(t)=\lambda \Phi(x)(t)$ for some $x \in \operatorname{cl}\left(X_{r}^{s}\right)$ and $0<\lambda<1$, then $x \in X_{r}^{s}$.

2. $\phi(r) \phi(s)<0$.

Then (2.1) has at least one solution $x \in c l\left(X_{r}^{s}\right)$.

Proof. For $\varphi \in X$, define $P \varphi(t):=\int_{0}^{t} \varphi(u) d u$ and

$$
K \varphi(t):=P \varphi(t)-\overline{P \varphi}+\left(\frac{T}{2}-t\right) \bar{\varphi} .
$$

In other words, $K \varphi$ is defined as the unique element $x \in X$ such that $x^{\prime}(t)=$ $\varphi(t)-\bar{\varphi}$ for all $t$ and $\bar{x}=0$.

Next define, for $\lambda \in[0,1]$, the compact operator $\mathcal{K}_{\lambda}: X \rightarrow X$ given by

$$
\mathcal{K}_{\lambda} x(t):=\bar{x}+\overline{\Phi(x)}+\lambda K \Phi(x)(t) .
$$

For $\lambda>0$, it is readily seen that

$$
x^{\prime}(t)=\lambda \Phi(x)(t) \text { for all } t \Longleftrightarrow x(t)=\mathcal{K}_{\lambda} x(t) \text { for all } t .
$$

Indeed, if $x \in X$ is such that $x^{\prime}(t)=\lambda \Phi(x)(t)$ for all $t$, then integration at both sides and the fact that $\lambda \neq 0$ yield $\overline{\Phi(x)}=0$. It follows that $x-\bar{x}=\lambda K \Phi(x)$ and hence $x=\bar{x}+\lambda K \Phi(x)=\bar{x}+\overline{\Phi(x)}+\lambda K \Phi(x)$. Conversely, if $x \in X$ satisfies the equality $x(t)=\mathcal{K}_{\lambda} \Phi(x)(t)$ for all $t$, then $x^{\prime}(t)=\lambda[K \Phi(x)]^{\prime}(t)=$ $\lambda[\Phi(x)(t)-\overline{\Phi(x)}]$. Moreover, as $\bar{x}=\overline{\mathcal{K}_{\lambda} \Phi(x)}=\bar{x}+\overline{\Phi(x)}$, we deduce that $\overline{\Phi(x)}=0$ and the claim is proven.

Thus, it suffices to verify that the function $\mathcal{F}_{\lambda}:=I d-\mathcal{K}_{\lambda}$ vanishes in $\operatorname{cl}\left(X_{r}^{s}\right)$ for $\lambda=1$. Assumption 1 implies that $\mathcal{F}_{\lambda}$ does not vanish on $\partial X_{r}^{s}$ for $0<\lambda<1$; on the other hand, observe that $\operatorname{Im}\left(\mathcal{K}_{0}\right) \subset \mathbb{R}$ and thus assumption 2 implies that $\mathcal{F}_{0}$ does not vanish on $\partial X_{r}^{s}$. Furthermore, if $\gamma \in \mathbb{R} \subset X$ then $\mathcal{F}_{0}(\gamma)=\gamma-[\bar{\gamma}+\overline{\Phi(\gamma)}]=-\phi(\gamma)$. It follows that

$$
\operatorname{deg}_{L S}\left(\mathcal{F}_{0}, X_{r}^{s}, 0\right)=-\operatorname{deg}_{B}(\phi,(r, s), 0)=\left\{\begin{array}{r}
1 \text { if } \phi(r)>0>\phi(s) \\
-1 \text { if } \phi(r)<0<\phi(s) .
\end{array}\right.
$$

From the homotopy invariance of the Leray-Schauder degree, we conclude that $\operatorname{deg}_{L S}\left(\mathcal{F}_{1}, X_{r}^{s}, 0\right)= \pm 1$ and the result follows.

In particular, the previous continuation theorem applies to the following problems with state-dependent delay:

$$
x^{\prime}(t)=F(t, x(t), x(t-\tau(t, x(t))))
$$

and the integro-differential equation

$$
x^{\prime}(t)=F\left(t, x(t), \int_{t-\tau(t, x(t))}^{t} h(s, x(s)) d s\right)
$$


where $\tau, h: \mathbb{R}^{2} \rightarrow \mathbb{R}$ and $F: \mathbb{R}^{3} \rightarrow \mathbb{R}$ are continuous and $T$-periodic in the first coordinate. Indeed, it suffices to consider the Nemitskii operators $\Phi: X \rightarrow X$ given by

$$
\Phi(x)(t):=F(t, x(t), x(t-\tau(t, x(t))))
$$

and

$$
\Phi(x)(t):=F\left(t, x(t), \int_{t-\tau(t, x(t))}^{t} h(s, x(s)) d s\right)
$$

respectively. The next section is devoted to the study of some specific cases of Eqs. (2.2) and (2.3).

\subsection{Applications to general equations with delay}

Motivated by the applications (see Appendix), let us firstly consider the following abstract problems:

$$
x^{\prime}(t)=-a(t)+h(t, x(t), x(t-\tau(t, x(t)))),
$$

and

$$
x^{\prime}(t)=-a(t) x(t)+g(t, x(t), x(t-\tau(t, x(t))))
$$

or, more generally,

$$
x^{\prime}(t)=-f(t, x(t))+g(t, x(t), x(t-\tau(t, x(t))))
$$

along with the corresponding "mirror" equations. Throughout this section we shall assume that $h: \mathbb{R}^{3} \rightarrow[0,+\infty), g: \mathbb{R} \times[0,+\infty)^{2} \rightarrow[0,+\infty)$ and $f, \tau$ : $\mathbb{R} \times[0,+\infty) \rightarrow[0,+\infty)$ are continuous and $T$-periodic in the first coordinate and that $a \in X$ satisfies $a(t)>0$ for all $t$. All the results in this section can be established analogously for their "mirror" versions. The following results should be considered just as general applications of the abstract continuation theorem, but they might be improved in some specific cases, if the nonlinearities satisfy for example certain local or global monotonicity conditions. For convenience, let us define the quantities $a_{\tau}(x):=\max _{t \in[0, T]} \int_{t-\tau(t, x)}^{t} a(s) d s, a_{T}:=$ $\int_{0}^{T} a(t) d t$ and $a_{0}:=\sup _{x \in \mathbb{R}} \min \left\{a_{\tau}(x), a_{T}\right\}$.

Theorem 2.2. Assume there exist constants $r<s$ such that

$$
[h(t, r, r+u)-a(t)] \cdot[h(t, s, s-v)-a(t)]<0
$$

for all $t \in \mathbb{R}$ and any $u, v$ such that $0 \leq u \leq a_{0}$ and $0 \leq v \leq a_{T}$. Then (2.4) admits at least one $T$-periodic solution in $X_{r}^{s}$.

Proof. In the setting of Theorem 2.1, observe that

$$
\phi(\gamma)=\frac{1}{T} \int_{0}^{T}[h(t, \gamma, \gamma)-a(t)] d t
$$

In particular, using (2.7) with $u=v=0$, it is clear that the functions $h(\cdot, r, r)-$ $a$ and $h(\cdot, s, s)-a$ do not vanish and have opposite signs; thus $\phi(r) \phi(s)<0$. Furthermore, if $x \in \operatorname{cl}\left(X_{r}^{s}\right)$ satisfies

$$
x^{\prime}(t)=\lambda[-a(t)+h(t, x(t), x(t-\tau(t, x(t))))]
$$


for some $\lambda \in(0,1]$, then $x^{\prime}(t) \geq-\lambda a(t) \geq-a(t)$ and hence $x\left(t_{1}\right)-x\left(t_{2}\right) \leq$ $\int_{t_{1}}^{t_{2}} a(t) d t$ for any $t_{1} \leq t_{2}$. As $x$ is $T$-periodic the previous inequality implies, in particular, that $x(t) \leq x_{\text {min }}+a_{T}$ for all $t$. Moreover, if $x$ reaches its absolute minimum at $t=\xi$, then $x(\xi-\tau(\xi, x(\xi))) \leq x_{\min }+a_{\tau}(x(\xi))$ and thus $x(\xi-\tau(\xi, x(\xi))) \leq x_{\text {min }}+a_{0}$. Suppose that $x_{\text {min }}=r$, then

$$
0=x^{\prime}(\xi)=\lambda[-a(\xi)+h(\xi, r, r+u)]
$$

for some $u$ such that $0 \leq u \leq a_{0}$, a contradiction. Similarly, $x_{\max } \neq s$ and the conclusion follows.

For model (2.5) we obtain the following statement.

Theorem 2.3. Assume there exist positive constants $\rho<\sigma$ such that

$$
\left[\frac{g(t, \rho, k \rho)}{\rho}-a(t)\right] \cdot\left[\frac{g(t, \sigma, \sigma / l)}{\sigma}-a(t)\right]<0
$$

for all $t \in \mathbb{R}$ and any $k, l$ such that $1 \leq k \leq e^{a_{0}}$ and $1 \leq l \leq e^{a_{T}}$. Then (2.5) admits at least one positive $T$-periodic solution in $X_{\rho}^{\sigma}$.

Proof. Set $y(t)=\ln (x(t))$, then Eq. (2.5) is equivalent to

$$
y^{\prime}=-a(t)+h\left(t, y(t), y\left(t-\tau\left(t, e^{y(t)}\right)\right)\right),
$$

where

$$
h(t, y, z):=\frac{g\left(t, e^{y}, e^{z}\right)}{e^{y}} .
$$

Thus, the proof follows from the previous theorem, taking $r:=\ln \rho$ and $s:=$ $\ln \sigma$.

The preceding theorem is readily adapted to model (2.6):

Theorem 2.4. Assume there exists $a \in X$ such that $f(t, x) \leq a(t) x$ for all $t$ and all $x \geq 0$. Further, assume there exist positive constants $\rho<\sigma$ such that

$$
[h(t, \rho, k \rho)-f(t, \rho)] \cdot[h(t, \sigma, \sigma / l)-f(t, \sigma)]<0
$$

for all $t \in \mathbb{R}$ and any $k, l$ such that $1 \leq k \leq e^{a_{0}}$ and $1 \leq l \leq e^{a_{T}}$. Then (2.6) admits at least one positive T-periodic solution in $X_{\rho}^{\sigma}$.

We may also consider the following integro-differential problem

$$
x^{\prime}(t)=-f(t, x(t))+g(t, x(t)) \int_{t-\tau(t, x(t))}^{t} h(s, x(s)) d s
$$

and its "mirror" version, with $\tau, f, g, h: \mathbb{R} \times[0,+\infty) \rightarrow[0,+\infty)$ continuous and $T$-periodic in the first coordinate. For convenience define, for each $\alpha \geq 0$ :

$$
h_{\min }(\alpha):=\min _{0 \leq t \leq T} h(t, \alpha), \quad h_{\max }(\alpha):=\max _{0 \leq t \leq T} h(t, \alpha) .
$$

Then a straightforward imitation of the preceding proofs yields: 
Theorem 2.5. Assume there exists $a \in X$ such that $f(t, x) \leq a(t) x$ for all $t$ and all $x \geq 0$. Further, assume there exist nonnegative constants $\rho<\sigma$ such that

$$
\tau(t, \rho) g(t, \rho) h_{\max }(k \rho)-f(t, \rho)<0<\tau(t, \sigma) g(t, \sigma) h_{\min }(\sigma / l)-f(t, \sigma)
$$

or

$$
\tau(t, \rho) g(t, \rho) h_{\min }(k \rho)-f(t, \rho)>0>\tau(t, \sigma) g(t, \sigma) h_{\max }(\sigma / l)-f(t, \sigma)
$$

for all $t \in \mathbb{R}$ and any $k, l$ such that $1 \leq k \leq e^{a_{0}}$ and $1 \leq l \leq e^{a_{T}}$. Then Eq. (2.8) admits at least one positive T-periodic solution in $X_{\rho}^{\sigma}$.

\subsection{Sublinear models}

In this section we shall focus on a special class of sublinear models. Consider

$$
x^{\prime}(t)=-a(t) x(t)+g(t, x(t-\tau(t, x(t)))),
$$

with $a \in X, \tau, g: \mathbb{R} \times[0,+\infty) \rightarrow[0,+\infty)$ continuous and $T$-periodic in the first coordinate, and define the limits $\gamma_{0, \infty} \in[0,+\infty]$ given by

$$
\gamma_{0}:=\liminf _{u \rightarrow 0^{+}} \min _{t \in[0, T]} \frac{g(t, u)}{u}, \quad \gamma_{\infty}:=\limsup _{u \rightarrow+\infty} \max _{t \in[0, T]} \frac{g(t, u)}{u} .
$$

In this setting, the sublinearity condition that shall be assumed throughout the section can be simply expressed as: $\gamma_{\infty}<\infty$. In other words, we shall assume that the function $g(t, \cdot)$ grows at most linearly, uniformly for $t \in \mathbb{R}$.

Our first result is a direct consequence of Theorem 2.3:

Theorem 2.6. Assume that

$$
\gamma_{\infty}<a(t)<\gamma_{0} \text { for all } t .
$$

Then Eq. (2.9) admits at least one positive T-periodic solution.

Proof. Since $\gamma_{\infty}<a_{m i n}$, there exists a constant $\sigma_{\infty}>0$ such that

$$
\frac{g(t, \tilde{\sigma})}{\tilde{\sigma}}<a_{\min } \quad \forall \tilde{\sigma} \geq \sigma_{\infty}, \forall t \in[0, T] .
$$

Similarly, there exists a constant $\rho_{0}>0$ such that

$$
\frac{g(t, \tilde{\rho})}{\tilde{\rho}}>a_{\max } \quad \forall \tilde{\rho} \in\left(0, \rho_{0}\right], \forall t \in[0, T] .
$$

Hence, it suffices to take arbitrary positive constants $\rho, \sigma$ satisfying

$$
\rho \leq \rho_{0} e^{-a_{0}}, \quad \sigma \geq \sigma_{\infty} e^{a_{T}}
$$

and apply Theorem 2.3.

In a similar fashion, a generalization of this result for a sublinear case of Eq. (2.6) can be obtained from Theorem 2.4.

It is worth noticing that the assumption in Theorem 2.6 implies, in particular, that $a(t)>0$ for all $t$. The next theorem for model (2.9) assumes a weaker condition on $a$, provided that the nonlinearity is strictly sublinear. In this case, the proof does not follow from Theorem 2.3, which is no longer valid when $a \ngtr 0$, so we shall introduce a direct fixed point argument.

For the reader's convenience, let us recall the well known Schaefer's Theorem: 
Theorem 2.7. [33] Let $\mathbb{E}$ be a Banach space and let $K: \mathbb{E} \rightarrow \mathbb{E}$ be a compact operator. Assume there exists a constant $M$ such that

$$
x=\lambda K x \text { for some } \lambda \in(0,1] \Rightarrow\|x\| \leq M .
$$

Then $K$ has at least one fixed point.

Remark 2.8. In some texts, Schaefer's theorem is known as Leray-Schauder fixed point theorem, although the latter is more general. Its proof can be easily deduced from the properties of the Leray-Schauder degree and it has the advantage of simplicity, in the sense that the assumptions do not involve the concept of degree. However, the degree method is more powerful, in the sense that it can be applied to some situations that are not covered by Schaefer's theorem.

Theorem 2.9. Assume that $\bar{a}>0, \gamma_{\infty}=0$ and

$$
g(t, 0) \neq 0 \text { for some } t
$$

or

$$
a(t)<\gamma_{0} \text { for all } t .
$$

Then (2.9) admits at least one positive T-periodic solution.

Proof. Since $\bar{a}>0$, for any $\varphi \in X$ the linear problem $x^{\prime}(t)+a(t) x(t)=\varphi(t)$ has a unique $T$-periodic solution. In particular, the open mapping theorem implies the existence of a constant $c$ such that $\|x\|_{\infty} \leq c\left\|x^{\prime}+a x\right\|_{\infty}$ for every $x \in X \cap C^{1}(\mathbb{R}, \mathbb{R})$. Thus, we may define $K: X \rightarrow X$ as the compact operator given by $K w(t):=x(t)$, where $x \in X$ is the unique $T$-periodic solution of the linear problem

$$
x^{\prime}(t)+a(t) x(t)=g(t,|w(t-\tau(t, x(t)))|) .
$$

We seek a positive fixed point of $K$. Let us firstly assume that (2.10) holds. We claim that if $x$ is a fixed point of $K$, then $x(t)>0$ for all $t$. Indeed, let $A(t):=\int_{0}^{t} a(s) d s$, then

$$
\left(e^{A} x\right)^{\prime}(t)=e^{A(t)} g(t,|x(t-\tau(t, x(t)))|) \geq 0 .
$$

Suppose that equality holds for all $t$, then $g(t,|x(t-\tau(t, x(t)))|)=0$ for all $t$ which, in turn, implies that $x \equiv 0$. Thus $g(\cdot, 0) \equiv 0$, a contradiction. Hence the previous inequality is strict for some $t$, and then integration on $[t, t+T]$ yields

$$
e^{A(t+T)} x(t+T)>e^{A(t)} x(t) .
$$

Due to the periodicity we deduce:

$$
e^{A(t)}\left(e^{A(T)}-1\right) x(t)>0
$$

and therefore $x(t)>0$ for all $t$. Thus, from Schaefer's Theorem, it suffices to establish the existence of a constant $M$ such that all the $T$-periodic solutions of the problem

$$
x^{\prime}(t)+a(t) x(t)=\lambda g(t,|x(t-\tau(t, x(t)))|)
$$


with $\lambda \in(0,1]$ satisfy the inequality $\|x\|_{\infty} \leq M$. As before, it is seen that $x(t)>0$ for all $t$, so it suffices to find a uniform bound for $x_{\max }$. With this aim, fix a constant $\varepsilon>0$ to be specified. Since $\gamma_{\infty}=0$, there exists a constant $k>0$ such that $g(t, u) \leq \varepsilon u+k$ for all $u \geq 0$. Then

$x_{\max }=\|x\|_{\infty} \leq c\left\|x^{\prime}+a x\right\|_{\infty}=c \lambda \max _{t \in[0, T]} g(t, x(t-\tau(t, x(t)))) \leq c\left(\varepsilon x_{\max }+k\right)$.

Thus, choosing $\varepsilon<\frac{1}{c}$ it follows that $x_{\max }<\frac{k}{1-\varepsilon c}:=M$.

Next, assume that (2.11) holds. Let $\rho_{0}>0$ be chosen as in Theorem 2.6 and fix a positive $\alpha_{0} \leq \rho_{0} e^{-\int_{0}^{T}|a(t)| d t}$. As $\alpha_{0}<\rho_{0}$ and $a_{\max }>0$, then $g\left(t, \alpha_{0}\right)>0$ for all $t$. Let us define

$$
\tilde{g}(t, u):= \begin{cases}g(t, u) & \text { if } u \geq \alpha_{0} \\ g\left(t, \alpha_{0}\right) & \text { if } u<\alpha_{0} .\end{cases}
$$

From the preceding case, we deduce the existence of a positive $T$-periodic solution $x$ of (2.9) with $\tilde{g}$ instead of $g$.

We claim that $x_{\text {min }} \geq \alpha_{0}$. Indeed, in first place let us observe that, as $x^{\prime}(t) \geq-a(t) x(t)$ then $x_{\text {min }} \geq e^{-\int_{0}^{T}|a(t)| d t} x_{\text {max }}$. Now suppose $x_{\text {min }}<\alpha_{0}$, then $x_{\max }<\rho_{0}$ and hence

$$
g(t, x(t-\tau(t, x(t))))>a_{\max } x(t-\tau(t, x(t))) \geq a(t) x(t-\tau(t, x(t)))
$$

for all $t$. Fix $t_{0}$ such that $x\left(t_{0}\right)=x_{\text {min }}$, then

$$
a\left(t_{0}\right) x_{\text {min }}=g\left(t_{0}, x\left(t_{0}-\tau\left(t_{0}, x_{\text {min }}\right)\right)\right) \geq 0
$$

and, in particular, $a\left(t_{0}\right) \geq 0$. Furthermore, $a\left(t_{0}\right) x_{\text {min }}=g\left(t_{0}, x\left(t_{0}-\tau\left(t_{0}, x_{\text {min }}\right)\right)\right)>a\left(t_{0}\right) x\left(t_{0}-\tau\left(t_{0}, x_{\text {min }}\right)\right) \geq a\left(t_{0}\right) x_{\text {min }}$, a contradiction. We conclude that $x_{\min } \geq \alpha_{0}$; this fact implies that

$$
\tilde{g}(t, x(t-\tau(t, x(t))))=g(t, x(t-\tau(t, x(t))))
$$

for all $t$ and the proof is complete.

A more general result may be obtained for model (2.6) as a straightforward imitation of the proof of Theorem 2.9.

\subsection{Monotone case: properties of the solutions}

In this section we shall study the behavior of the solutions of (2.9) with a state-independent delay, namely the equation

$$
x^{\prime}(t)=-a(t) x(t)+g(t, x(t-\tau(t)))
$$

under the following conditions:

1. $a$ and $\tau$ are continuous and $T$-periodic functions with $\tau(t) \geq 0$ for all $t$ and $\bar{a}>0$.

2. $g: \mathbb{R} \times[0,+\infty) \rightarrow(0,+\infty)$ is continuous, $T$-periodic in the first coordinate and non-increasing in the second coordinate.

We recall from Theorem 2.9 that these conditions imply the existence of at least one positive $T$-periodic solution. Also, it is easy to check that solutions of the initial value problem with positive initial data $\varphi \in C\left(\left[-\tau_{\max }, 0\right], \mathbb{R}\right)$ are globally defined and positive for all $t$. 
Proposition 2.10. Let $x, y>0$ be solutions of (2.12). If there exists a constant $t_{0}$ such that $x(t) \geq y(t)$ for all $t \geq t_{0}$, then $\lim _{t \rightarrow+\infty}[x(t)-y(t)]=0$.

Proof. Let $w(t)=x(t)-y(t)$, then

$$
w^{\prime}(t)+a(t) w(t)=g(t, x(t-\tau(t)))-g(t, y(t-\tau(t))) \leq 0
$$

for $t-\tau_{\max } \geq t_{0}$. Setting $A(t):=\int_{0}^{t} a(s) d s$, yields

$$
\left(e^{A} w\right)^{\prime}(t) \leq 0
$$

for $t \geq t_{0}+\tau_{\max }$. In particular,

$$
e^{A(t)} w(t) \leq e^{A\left(t_{0}+\tau_{\max }\right)} w\left(t_{0}+\tau_{\max }\right),
$$

that is

$$
w(t) \leq e^{-\int_{t_{0}+\tau_{\max }}^{t} a(s) d s} w\left(t_{0}+\tau_{\max }\right) .
$$

Since $\bar{a}>0$, we deduce from periodicity that $\int_{t_{0}+\tau_{\max }}^{t} a(s) d s \rightarrow+\infty$ as $t \rightarrow$ $+\infty$ and the conclusion follows.

Proposition 2.11. If $x, y>0$ are T-periodic solutions of (2.12), then there exist at least two points $t_{1}, t_{2} \in[0, T)$ such that $x\left(t_{j}\right)=y\left(t_{j}\right)$.

Proof. From Proposition 2.10, if, for example, $x(t) \geq y(t)$ for all $t$, then $\lim _{t \rightarrow+\infty}[x(t)-y(t)]=0$, therefore $x(t) \equiv y(t)$. The conclusion follows from periodicity.

Proposition 2.12. Assume $a(t)>0$ for all $t$ and define

$$
M:=\max _{t \in[0, T]} \int_{t}^{t+\tau_{\max }} g(s, 0) d s .
$$

Then for any given positive solutions $x, y$ of (2.12) there exists a constant $t_{0}$ such that $|x(t)-y(t)| \leq M$ for all $t \geq t_{0}$. In particular, positive $T$-periodic solutions of (2.12) are stable.

Proof. Let $x \neq y$ be two positive solutions of (2.12). If the graph of $x$ intersects the graph of $y$ at most finitely many times, we deduce from Proposition 2.10 that $\lim _{t \rightarrow+\infty}[x(t)-y(t)]=0$ and the result follows. Next, suppose that the graph of $x$ intersects the graph of $y$ infinitely many times and let $t_{0}<t_{1}$ be such that $w(t):=x(t)-y(t)>0$ on $\left(t_{0}, t_{1}\right)$ and $w\left(t_{j}\right)=0$. In first place, as $g$ is nonincreasing on its second coordinate and positive we deduce that

$$
w^{\prime}(t)+a(t) w(t)=g(t, x(t-\tau(t)))-g(t, y(t-\tau(t)))<g(t, 0),
$$

which implies

$$
w(t)<\int_{t_{0}}^{t} g(s, 0) e^{-\int_{s}^{t} a(r) d r} d s<\int_{t_{0}}^{t} g(s, 0) d s
$$

for all $t \geq t_{0}$. Let $\eta \in\left(t_{0}, t_{1}\right)$ be such that $w(\eta)=\max _{t_{0} \leq t \leq t_{1}} w(t)$. We claim that $\eta \leq t_{0}+\tau_{\max }$. Indeed, if $t_{0}+\tau_{\max } \geq t_{1}$ then the result is obvious; 
otherwise, for every $t \in\left(t_{0}+\tau_{\max }, t_{1}\right]$ we have that $x(t-\tau(t))>y(t-\tau(t))$ and consequently $w^{\prime}(t)+a(t) w(t) \leq 0$. As before,

$$
w(t) \leq e^{-\int_{t_{0}+\tau_{\max }}^{t} a(s) d s} w\left(t_{0}+\tau_{\max }\right)<w\left(t_{0}+\tau_{\max }\right) \leq w(\eta)
$$

for $t \in\left(t_{0}+\tau_{\max }, t_{1}\right]$ and the claim is proven. Hence,

$$
\max _{t_{0} \leq t \leq t_{1}} w(t) \leq \max _{t_{0} \leq t \leq t_{0}+\tau_{\max }} w(t) \leq \int_{t_{0}}^{t_{0}+\tau_{\max }} g(s, 0) d s \leq M .
$$

\section{Examples and discussion}

In this section we shall discuss and give some examples concerning the results in Sects. 2.2 and 2.3 and the applications to the different models presented in the Appendix.

In first place, observe that Theorems $2.2,2.3,2.4$ and 2.5 can be readily adapted to a model with a finite number of delays $\tau_{1}, \ldots, \tau_{N}: \mathbb{R}^{2} \rightarrow[0,+\infty)$ continuous and $T$-periodic in the first coordinate. For example, existence of positive $T$-periodic solutions of model (4.1) follows easily, provided that $a>0$ and $b_{i}>0$ for $i=1, \ldots, N$ continuous and $T$-periodic. Direct application of Theorem 2.2 shows the existence of a positive $T$-periodic solution for models (4.8) and (4.10), and also for (4.5) and (4.11), provided that $b(t)>a(t)>0$ for all $t$ (see also [34]). Another application of Theorem 2.2 is given in the next example.

Example. Consider the problems

$$
x^{\prime}(t)=-a(t)+b(t) x^{k}(t) \frac{x^{m}(t-\tau(t))}{c(t)+x^{n}(t-\tau(t))}
$$

and

$$
x^{\prime}(t)=a(t)-b(t) x^{k}(t) \frac{x^{m}(t-\tau(t))}{c(t)+x^{n}(t-\tau(t))}
$$

with $a, b, c>0$ continuous $T$-periodic functions, $m, n \geq 0$ and $k>0$. Then:

1. If $k+m>n$, then there exists at least one positive $T$-periodic solution.

2. If $k+m=n$, then there exists at least one positive $T$-periodic solution, provided that $b(t)>a(t)$ for all $t$.

3. If $k+m<n$, then there exist at least two positive $T$-periodic solutions, provided that $a(t)<M(t) b(t)$ for all $t$, where

$$
M:=\max _{|w| \leq a_{T} \leq s} \frac{s^{k}(s+w)^{m}}{c(t)+(s+w)^{n}} .
$$

In particular, model (4.9) is contained in case 1 with $k=1$ and $m=n$. This case also contains the equation considered in [8], which is a "mirror" equation for (4.9). Model (4.12) is included into the three previous cases with $k=1, m=0$ and $n<1, n=1, n>1$ respectively.

The following two examples are direct applications of Theorem 2.3. 
Example. The Michaelis-Menten Model (4.14)

$$
x^{\prime}(t)=x(t)\left[a(t)-\frac{b(t) x(t-\tau(t))}{1+c(t) x(t-\tau(t))}\right]
$$

admits at least one $T$-periodic positive solution, provided that $a, b, c>0$ are continuous and $T$-periodic and $b(t)>a(t)$ for all $t$. Similar conclusions hold for models (4.2) and (4.3) for arbitrary positive continuous $T$-periodic functions $a, b, c$ and for (4.13), provided that $a, b$ and $c$ are positive $T$-periodic functions and $\ln c(t)<\frac{a(t)}{b(t)}$ for all $t$.

Example. Consider the equation

$$
x^{\prime}(t)=-\alpha(t) x(t)-\frac{\beta(t) x(t)}{A(t)+x^{n}(t)}+2 e^{-\delta(t) \tau} \frac{\beta(t) x(t-\tau)}{A(t)+x^{n}(t-\tau)}
$$

with $\alpha, \beta, \delta, A: \mathbb{R} \rightarrow(0,+\infty)$ continuous and $T$-periodic and $\tau>0$ a constant. Set $f(t, x):=\alpha(t) x+\frac{\beta(t) x}{A(t)+x^{n}}$ and $a(t):=\alpha(t)+\frac{\beta(t)}{A(t)}$, then by Theorem 2.4 existence of a positive $T$-periodic solution follows under the condition

$$
2 e^{-\delta(t) \tau}>\frac{\alpha(t) A(t)}{\beta(t)}+1
$$

for all $t$. In particular, (3.2) implies that $\beta(t)>\alpha(t) A(t)$ for all $t$.

This equation was studied in [30] for

$$
\alpha \equiv \alpha_{0}, \quad \beta \equiv \beta_{0}, \quad A \equiv 1, \quad \delta \equiv \delta_{0} .
$$

Note that the condition

$$
\tau<-\frac{1}{\delta_{0}} \ln \frac{\alpha_{0}+\beta_{0}}{2 \beta_{0}},
$$

required in [30, p. 169] for the existence of a positive equilibrium $x_{*}$, is exactly the same as (3.2) applied to this particular case. Using the contraction mapping theorem, the authors prove, for some appropriate $\eta>0$, that if $n$ is large enough then there exists a unique positive $T$-periodic solution $x$ (for some period $T$ ) such that $x(t) \geq 1+\eta$ for $t \in[-\tau, 0]$. As $x_{*} \rightarrow 1$ for $n \rightarrow \infty$, it follows that $x$ is non-constant. This shows, in particular, that the solution obtained as an application of Theorem 2.3 is not unique. The problem of proving uniqueness or multiplicity of positive $T$-periodic solutions for arbitrary $n$ remains open.

The next example shows that Theorem 2.4 can be improved if one takes advantage of the monotonicity properties of the nonlinearity.

Example. Consider the following Nicholson's blowflies model with a nonlinear density-dependent mortality term, introduced in [3]:

$$
x^{\prime}(t)=-\frac{a(t) x(t)}{b(t)+x(t)}+c(t) x(t-\tau(t, x(t))) e^{-d(t) x(t-\tau(t, x(t)))}
$$

with $a, b, c, d>0$ continuous and $T$-periodic and $\tau: \mathbb{R} \times,+\infty) \rightarrow[0,+\infty)$ continuous and $T$-periodic in its first coordinate. Here, a sufficient condition (not 
deduced from Theorem 2.4) for the existence of positive $T$-periodic solutions is that $a(t)<b(t) c(t)$ for all $t$. Indeed, in the setting of Theorem 2.1 we have

$$
\phi(\gamma)=\frac{1}{T} \int_{0}^{T}\left[c(t) \gamma e^{-d(t) \gamma}-\frac{\gamma a(t)}{b(t)+\gamma}\right] d t
$$

so $\phi(s)<0<\phi(r)$ for $s \gg 0$ and $0<r \ll 1$. Moreover, if

$$
x^{\prime}(t)=\lambda\left(-\frac{a(t) x(t)}{b(t)+x(t)}+c(t) x(t-\tau(t, x(t))) e^{-d(t) x(t-\tau(t, x(t)))}\right)
$$

for some $0<\lambda<1$ then $x^{\prime}(t)>-a(t)$ and hence $x_{\max }-x_{\min }<\int_{0}^{T} a(t) d t$. If $x(\eta)=x_{\max }=s$ then

$$
\frac{a(\eta) s}{b(\eta)+s}=c(\eta)(s-v) e^{-d(\eta)(s-v)}
$$

for some $v$ such that $0 \leq v \leq \int_{0}^{T} a(t) d t$. This yields a contradiction when $s \gg 0$. On the other hand, if $x(\xi)=x_{\text {min }}=r>0$ then

$$
x(\xi-\tau(\xi, x(\xi))) e^{-d(\xi) x(\xi-\tau(\xi, x(\xi)))} \geq r e^{-d(\xi) r}
$$

when $r$ is small enough. This implies

$$
\frac{a(\xi)}{b(\xi)+r} \geq c(\xi) e^{-d(\xi) r},
$$

a contradiction for $r \ll 1$.

Next, we will illustrate an application of Theorem 2.5.

Example. Consider the Nicholson integro-differential Eq. (4.6)

$$
x^{\prime}(t)=-a(t) x(t)+b(t) \int_{t-\tau(t)}^{t} c(s) x(s) e^{-d(s) x(s)} d s
$$

for some positive, continuous and $T$-periodic functions $a, b, c, d$ and $\tau$. If

$$
c_{\text {min }}>\frac{a(t)}{\tau(t) b(t)} \text { for all } t,
$$

then the second condition in Theorem 2.5 is fulfilled. Indeed, here $f(t, x)=$ $a(t) x, g(t, x)=b(t)$ and $h(t, x)=c(t) x e^{-d(t) x}$ and

$$
\begin{array}{r}
\tau(t) g(t, \rho) h_{\min }(k \rho)-f(t, \rho) \geq\left(\tau(t) b(t) c_{\min } e^{-d_{\max } k \rho}-a(t)\right) \rho>0 \\
\tau(t) g(t, \sigma) h_{\max }(\sigma / k)-f(t, \sigma) \leq\left(\tau(t) b(t) c_{\max } e^{-d_{\min } \sigma / k}-a(t)\right) \sigma<0
\end{array}
$$

if the positive constants $\rho, \sigma$ satisfy $\rho \ll 1, \sigma \gg 0$. Similar results may be easily obtained for model (4.4).

The techniques developed in the present paper can be also regarded as a tool to shorten existing proofs. For example, the assumption of Theorem 2.3 is fulfilled if $g$ satisfies the condition $\left(H_{1}\right)$ or $\left(H_{2}\right)$ in [34, Theorem 2.1] (see Sect. 1 of the present paper). 
A less trivial example is the main theorem in [23] for the state-dependent model

$$
x^{\prime}(t)=-F(t, x(t-\tau(t, x(t))))
$$

with $F, \tau: \mathbb{R}^{2} \rightarrow \mathbb{R}$ continuous and $T$-periodic in the first coordinate, which establishes: if there exists a constant $B>0$ such that $x F(t, x)>0$ for all $t, x \in \mathbb{R}$ with $|x|>B$, and $F$ is either bounded from below or from above, then the problem has at least one $T$-periodic solution. This result can be seen as an immediate consequence of Theorem 2.2: indeed, if for example $F(t, x) \geq-M$ for all $t, x \in \mathbb{R}$, then it suffices to take $a \equiv M$ and $h(t, x(t-\tau))=$ $M+F(t, x(t-\tau))$ and condition (2.7) is satisfied for arbitrary $r<-(B+M)$, $s>B+M$.

In the same way, we may obtain a shorter proof of the result in [42] (mentioned in the introduction of the present paper) for the model

$$
x^{\prime}(t)=-a(t) x(t)+\lambda b(t) g(x(t-\tau(t)))
$$

with continuous $T$-periodic $a, b>0$ and continuous $g:[0,+\infty) \rightarrow(0,+\infty)$ nondecreasing and superlinear, namely $\frac{g(u)}{u} \rightarrow+\infty$ as $u \rightarrow+\infty$. Indeed, it suffices, as in Theorem 2.9, to consider the problem

$$
x^{\prime}(t)=-a(t) x(t)+\lambda b(t) g(|x(t-\tau(t))|) .
$$

Firstly, observe that if the problem has no positive $T$-periodic solutions for some $\lambda_{*}$, then, by Theorem 2.7, it has at least one positive $T$-periodic solution for some $\hat{\lambda}<\lambda_{*}$. Nonexistence for large values of $\lambda$ is immediate since $\frac{g(u)}{u}$ is bounded from below: if $x$ is a positive $T$-periodic solution, then $\frac{x_{\max }}{x_{\min }} \leq e^{a_{T}}$ and hence

$$
(\ln x)^{\prime}(t)+a(t)=\lambda b(t) \frac{g(x(t-\tau(t)))}{x(t-\tau(t))} \frac{x(t-\tau(t))}{x(t)} \geq \lambda b(t) e^{-T \bar{a}} \min _{u \geq 0} \frac{g(u)}{u} .
$$

The latter implies that $\lambda \bar{b} \min _{u \geq 0} \frac{g(u)}{u}<\bar{a} e^{T \bar{a}}$. Finally, if $\hat{x}$ is a positive $T$-periodic solution for some $\hat{\lambda}$ and $\lambda \in(0, \hat{\lambda})$, then it is easy to verify that the operator $K$ defined by $K w(t):=x(t)$, where $x$ is the unique $T$-periodic solution of the linear problem $x^{\prime}(t)+a(t) x(t)=\lambda b(t) g(|w(t-\tau(t))|)$ maps the set $\{x \in X: 0 \leq x(t) \leq \hat{x}(t)$ for all $t\}$ into itself, and hence, by Schauder theorem (see e.g. [27]), it has a fixed point. Thus, there exists $\lambda_{*}>0$ such that the problem has at least one positive $T$-periodic solution for $\lambda \in\left(0, \lambda_{*}\right)$ and no positive $T$-periodic solutions for $\lambda>\lambda_{*}$.

As a final remark, it is worth to observe that Theorems 2.2, 2.3 and 2.4 are valid for an arbitrary delay, even for $\tau \equiv 0$. However, this is not necessarily true if we allow the equations to depend on $\tau$. This is obviously the case of Theorem 2.5. Also, we may consider for example the modified problem (2.4):

$$
x^{\prime}(t)=-a(t)+\tau h(t, x(t), x(t-\tau))
$$

with $a$ and $h$ as in Sect. 2.2. Similarly to Theorem 2.2, existence of $T$-periodic solutions is proven if

$$
[\tau h(t, r, r+u)-a(t)] \cdot[\tau h(t, s, s-v)-a(t)]<0
$$


for some $r<s$, for all $t \in \mathbb{R}$ and $0 \leq u, v \leq a_{0}$. When $\tau=0$, the problem has no $T$-periodic solutions and the previous condition clearly fails.

In contrast with these examples, some problems may have no solutions when $\tau$ is large. For example, condition (3.2) ensures that, if $\alpha(t) A(t)<\beta(t)$ for all $t$, then problem (3.1) has at least one positive $T$-periodic solution for any $\tau \in\left[0, \tau_{*}\right)$, where

$$
\tau_{*}:=\left(\frac{1}{\delta} \ln \frac{2 \beta}{\alpha A+\beta}\right)_{\min } .
$$

On the other hand, the periodic problem has no positive solutions if $\tau$ is large. Indeed, if $x$ is such a solution, then setting $a(t):=\alpha(t)+\frac{\beta(t)}{A(t)}$ it is seen that $x^{\prime}(t)>-a(t) x(t)$ and hence $x_{\max }<x_{\min } e^{a_{T}}$. If $x(\eta)=x_{\max }$, then

$$
\alpha(\eta)=\frac{\beta(\eta) x(\eta-\tau)}{A(\eta)+x^{n}(\eta-\tau)}\left(2 e^{-\delta(\eta) \tau} \frac{x(\eta-\tau)}{x_{\max }}-\frac{A(\eta)+x^{n}(\eta-\tau)}{A(\eta)+x_{\max }^{n}}\right)
$$

and we obtain:

$$
\alpha(\eta)<\frac{\beta(\eta) x(\eta-\tau)}{A(\eta)+x^{n}(\eta-\tau)}\left(2 e^{-\delta(\eta) \tau}-e^{-n T \bar{a}}\right) .
$$

This implies that the problem has no $T$-periodic positive solutions when

$$
\tau \geq \frac{\ln 2+n T \bar{a}}{\delta_{\min }}
$$

Likewise, the results of Sect. 2.4 hold when $\tau \equiv 0$, although they become trivial: in this case, it is immediate that trajectories of the ordinary differential Eq. (2.12) do not intersect; thus, the positive $T$-periodic solution obtained from Theorem 2.9 is unique and globally asymptotically stable. The situation is different when $\tau \not \equiv 0$. For example, consider the particular case of Eq. (4.8) with $a \equiv \frac{1}{e}, b=c \equiv 1$ and $\tau \equiv 2 e$ :

$$
x^{\prime}(t)+\frac{x(t)}{e}=e^{-x(t-2 e)} .
$$

This equation has an equilibrium $x^{*}=1$; however, if $x$ is a solution with $x(0)=2$ and $x(t) \geq 2$ for $t \leq 0$, then $x(t-2 e) \geq 2$ for $t \leq 2 e$. Suppose that the graph of $x$ does not cross the equilibrium, then $x(t)>1$ for all $t$ and $x^{\prime}(t)<\frac{1-e}{e^{2}}$ for $0 \leq t \leq 2 e$. This implies $x(2 e)<\frac{2}{e}<1$, a contradiction.

\section{Appendix: basic scalar models of population dynamics}

Logistic models:

$$
\begin{aligned}
& \dot{x}=x(t)\left[a(t)-\sum_{i}^{n} b_{i}(t) x\left(t-\tau_{i}(t)\right)\right] \\
& \dot{x}=a(t) x(t)\left[1-\left(\frac{x(t-\tau(t))}{b(t)}\right)^{\theta}\right] \\
& \dot{x}=x(t)[a(t)-b(t) x(t)+c(t) x(t-\tau(t))]
\end{aligned}
$$




$$
\dot{x}=x(t)\left(a(t)-b(t) \int_{t-\tau(t)}^{t} c(s) x(s) d s\right)
$$

Nicholson models:

$$
\begin{aligned}
& \dot{x}=-a(t) x(t)+b(t) x(t-\tau(t)) e^{-c(t) x(t-\tau(t))} \\
& \dot{x}=-a(t) x(t)+b(t) \int_{t-\tau(t)}^{t} c(s) x(s) e^{-d(s) x(s)} d s
\end{aligned}
$$

Lasota-Wazewska model.

$$
\dot{x}=-a(t) x(t)+c(t) e^{-b(t) x(t-\tau(t))}
$$

Mackey-Glass models:

$$
\begin{aligned}
& \dot{x}=a(t)-b(t) \frac{x(t) x^{m}(t-\tau(t))}{1+x^{n}(t-\tau(t))} \\
& \dot{x}=-a(t) x(t)+\frac{b(t)}{1+x^{n}(t-\tau(t))} \\
& \dot{x}=-a(t) x(t)+\frac{b(t) x(t-\tau(t))}{1+x^{n}(t-\tau(t))} \\
& \dot{x}=-a(t) x(t)+\frac{b(t) x(t)}{1+x^{n}(t-\tau(t))}
\end{aligned}
$$

Gompertz (Fox production) model.

$$
\dot{x}=-a(t) x(t)+b(t) x(t) \ln \frac{c(t)}{x(t-\tau(t))}
$$

Michaelis-Menten model.

$$
\dot{x}=x(t)\left[a(t)-\frac{b(t) x(t-\tau(t))}{1+c(t) x(t-\tau(t))}\right]
$$

\section{Acknowledgments}

We are grateful to reviewers for their helpful comments that led to an improvement of this manuscript.

\section{References}

[1] Amster, P., Deboli, A.: Existence of positive image-periodic solutions of a generalized Nicholsons blowflies model with a nonlinear harvesting term. Appl. Math. Lett. 25, 1203-1207 (2012)

[2] Berezansky, L., Idels, L.: Periodic Fox harvesting models with delay. Appl. Math. Comput. 195, 142-153 (2008)

[3] Berezansky, L., Braverman, E., Idels, L.: Nicholsons blowflies differential equations revisited: main results and open problems. Appl. Math. Model. 34(6), 14051417 (2010) 
[4] Berezansky, L., Braverman, E., Idels, L.: Mackey-Glass model of hematopoiesis with monotone feedback revisited. Appl. Math. Comput. 219, 4892-4907 (2013)

[5] Berezansky, L., Braverman, E., Idels, L.: Mackey-Glass model of respiratory dynamics: review and new results. Nonlinear Anal. TMA 75, 6034-6052 (2012)

[6] Berezansky, L.; Braverman, E.; Idels, L.: The Mackey-Glass model of hematopoiesis with non-monotone feedback: stability, oscillation and control. Appl. Math. Comput. (2013) (in print)

[7] Chen, Y.: The existence of periodic solutions of the equation $x^{\prime}(t)=$ $-f(x(t), x(t-\tau))$. J. Math. Anal. Appl. 163, 227-237 (1992)

[8] Chen, Y., Huang, L.: Existence and global attractivity of a positive periodic solution of a delayed periodic respiration model. Comput. Math. Appl. 49, 677$687(2005)$

[9] Chen, Y.: Periodic solutions of delayed periodic Nicholson's blowflies models. Can. Appl. Math. Q. 11, 23-28 (2003)

[10] Cheng, S., Zhang, G.: Existence of positive periodic solutions for non-autonomous functional differential equations. EJDE 59, 1-8 (2001)

[11] Freedman, H., Wu, J.: Periodic solutions of single-species models with periodic delay. SIAM J. Math. Anal. 23, 689-701 (1992)

[12] Gaines, R., Mawhin, J.: Coincidence degree and nonlinear differential equations. Springer, Berlin (1977)

[13] Graef, J., Padhi, S., Pati, S.: Periodic solutions of some models with strong Allee effects. Nonlin. Anal. RWA 13, 569-581 (2012)

[14] Graef, J., Kon, L.: Existence of multiple periodic solutions for first order functional differential equations. Math. Comp. Model. 54, 2962-2968 (2011)

[15] Han, F., Wang, Q.: Existence of multiple positive periodic solutions for differential equation with state-dependent delays. J. Math. Anal. Appl. 324, 908$920(2006)$

[16] Hartung, F., Krisztin, T., Walther, H., Wu, J.: Functional differential equations with state-dependent delay: theory and applications. In: Canada, A., Drabek, P., Fonda, A. (eds.) Handbook of Differential Equations: Ordinary Differential Equations, vol. 3, pp. 435-545. Elsevier, North-Holand (2006)

[17] Hou, X., Duan, L., Huang, Z.: Permanence and periodic solutions for a class of delay Nicholsons blowflies models. Appl. Math. Model. 3, 1537-1544 (2013)

[18] Kang, S., Zhang, G.: Existence of nontrivial periodic solutions for first order functional differential equations. Appl. Math. Lett. 18, 101-107 (2005)

[19] Kuang, Y.: Delay Differential Equations with Applications in Population Dynamics. Academic Press, Boston (1993)

[20] Li, J., Du, C.: Existence of positive periodic solutions for a generalized Nicholson's blowflies model. Comput. Appl. Math. 221, 226-233 (2008) 
[21] Li, J., Wang, Z.: Existence and global attractivity of positive periodic solutions of a survival model of red blood cells. Comput. Math. Appl. 50, 41-47 (2005)

[22] Li, Y., Zhu, L.: Positive periodic solutions of nonlinear functional differential equations. Appl. Math. Comput. 156, 329-339 (2004)

[23] Li, Y., Kuang, Y.: Periodic solutions in periodic state-dependent delay equations and population models. Proc. Amer. Math. Soc. 130, 1345-1353 (2002)

[24] Liu, X., Li, W.: Existence and uniqueness of positive periodic solutions of functional differential equations. J. Math. Anal. Appl. 293, 28-39 (2004)

[25] Liu, G., Yan, J., Zhang, F.: Existence and global attractivity of unique positive periodic solution for a Lasota-Wazewska model. Nonlinear Anal. 64, 1737$1746(2006)$

[26] Liu, G., Yan, J., Zhang, F.: Existence and global attractivity of unique positive periodic solution for a model of hematopoiesis. J. Math. Anal. Appl. 334, 157$171(2007)$

[27] Lloyd, N.: Degree Theory. Cambridge University Press, Cambridge (1978)

[28] Ma, R., Chen, R., Chen, T.: Existence of positive periodic solutions of nonlinear first-order delayed differential equations. J. Math. Anal. Appl. 384, 527$535(2011)$

[29] Mackey, M., Glass, L.: Oscillations and chaos in phycological control systems. Sciences 197, 287-289 (1987)

[30] Mackey, M., Ou, C., PujoMenjouet, L., Wu, J.: Periodic oscillations of blood cell populations in chronic myelogenous leukemia. SIAM J. Math. Anal. 381, 166-187 (2006)

[31] Mallet-Paret, J., Nussbaum, R.: Stability of periodic solutions of state-dependent delay-differential equations. J. Diff. Equ. 250, 4085-4103 (2011)

[32] Nan, Z., Chen, W., Li, L.: Positive periodic solutions for a class of delay differential equations. Appl. Math. Comput. 218, 4647-4650 (2011)

[33] Schaefer, H.: Über die Methode der a priori-Schranken. Math. Ann. 129, 415$416(1955)$

[34] Wan, A., Jiang, D., Xu, X.: A new existence theory for positive periodic solutions to functional differential equations. Comput. Math. Appl. 47, 1257-1262 (2004)

[35] Wan, A., Jiang, D.: Existence of positive periodic solutions for functional differential equations. Kyush J. Math. 56, 193-202 (2002)

[36] Wang, H.: Positive periodic solutions of functional differential equations. J. Diff. Equ. 202, 354-366 (2004)

[37] Wu, Y.: Existence of positive periodic solutions for a functional differential equation with a parameter. Nonlinear Anal. 68, 1954-1962 (2008) 
[38] Wu, X., Li, J., Zhou, H.: A necessary and sufficient condition for the existence of positive periodic solutions of a model of hematopoiesis. Comput. Math. Appl. 54, 840-849 (2007)

[39] Wu, K., Wu, X.: Multiplicity results of periodic solutions for systems of first order delay differential equation. Appl. Math. Comput. 218, 1765-1773 (2011)

[40] Ye, D., Fan, M., Wang, H.: Periodic solutions for scalar functional differential equations. Nonlinear Anal. 62, 1157-1181 (2005)

[41] Zhang, W., Zhu, D., Bi, P.: Existence of periodic solutions of a scalar functional differential equation via a fixed point theorem. Math. Comput. Model. 46, 718$729(2007)$

[42] Zhang, G., Cheng, S.: Positive periodic solutions of nonautonomous functional differential equations depending on a parameter. Abstr. Appl. Anal. 7, 279$286(2002)$

[43] Zeng, Z., Bi, L., Fan, M.: Existence of multiple positive periodic solutions for functional differential equations. J. Math. Anal. Appl. 325, 1378-1389 (2007)

Pablo Amster

Universidad de Buenos Aires and CONICET

Buenos Aires

Argentina

e-mail: pamster@dm.uba.ar

Lev Idels

Department of Mathematics

Vancouver Island University

900 Fifth St.

Nanaimo

BC V9S5S5

Canada

e-mail: lev.idels@viu.ca

Received: 19 July 2012.

Accepted: 19 January 2013. 